KUNS-1404

$\mathrm{HE}(\mathrm{TH}) 96 / 07$

hep-th/9608128

\title{
Causality in Covariant String Field Theory
}

\author{
Hiroyuki Hata*† and Hajime Oda ${ }^{\ddagger}$ \\ Department of Physics, Kyoto University \\ Kyoto 606-01, Japan
}

August, 1996

\begin{abstract}
Causality is studied in the covariant formulation of free string field theory (SFT). We find that, though the string field in the covariant formulation is a functional of the ghost coordinates as well as the space-time coordinate and the latter contains the time-like oscillators with negative norm, the condition for the commutator of two open string fields to vanish is simply given by $\int_{0}^{\pi} d \sigma\left(\Delta X^{\mu}(\sigma)\right)^{2}>0$, which is the same condition as in the light-cone gauge SFT. For closed SFT, the corresponding condition is given in a form which is manifestly invariant under the rigid shifts of the $\sigma$ parameters of the two string fields.
\end{abstract}

${ }^{*}$ e-mail address: hata@gauge.scphys.kyoto-u.ac.jp

${ }^{\dagger}$ Supported in part by Grant-in-Aid for Scientific Research from Ministry of Education, Science and Culture (\#07640394).

${ }_{\ddagger}^{\ddagger}$ e-mail address: oda@gauge.scphys.kyoto-u.ac.jp 


\section{Introduction}

Study of causal properties of string theory is an interesting and important subject since string is an extended object and could behave quite differently from point particle theories. In fact, Martinec [1] examined causality in free (non-interacting) open SFT using the light-cone gauge formulation [2, 3, 团. He found that the commutator of two string fields with string coordinates $X^{\mu}(\sigma)$ and $\widetilde{X}^{\mu}(\sigma)$, respectively, vanishes if the condition

$$
\int_{0}^{\pi} d \sigma\left(X^{\mu}(\sigma)-\widetilde{X}^{\mu}(\sigma)\right)^{2}>0
$$

is satisfied. . This condition may look unintuitive since it does not require that all points on $X^{\mu}(\sigma)$ are space-like separated from all points on $\widetilde{X}^{\mu}(\sigma)$. Causality in free light-cone SFT was further studied by Lowe [5], and the analysis in the interacting SFT was carried out in ref. [6].

The purpose of this note is to reexamine the causality in free SFT on the basis of its covariant formulation [7, 8], which is expected to be more suited to the study of the spacetime properties of string theory than the light-cone gauge formulation. Besides the choice of the time variable for quantization, the main differences between the covariant formulation and the light-cone gauge one are that the string field in the former is a functional of the ghost string coordinates as well as the space-time string coordinate $X^{\mu}(\sigma)$ and that the latter contains time-like oscillator modes with negative norm. In spite of these differences, we find that the commutator of two string fields in the covariant formulation vanishes when the same condition as (1.1) is satisfied in the open SFT case. We also examine the closed SFT, and find that the condition for the closed string field commutator to vanish is given, instead of (1.1), by

$$
\min _{0 \leq \theta \leq 2 \pi} \int_{0}^{2 \pi} d \sigma(X(\sigma+\theta)-\widetilde{X}(\sigma))^{2}>0
$$

which is manifestly invariant under the rigid shift of each $\sigma$ parameter of $X^{\mu}(\sigma)$ and $\widetilde{X}^{\mu}(\sigma)$.

\section{Commutator of string fields}

String field in the covariant formulation of SFT is a functional of the ghost and the anti-ghost coordinates, $\Theta(\sigma)$ and $\bar{\Theta}(\sigma)$, as well as the space-time coordinate $X^{\mu}(\sigma)$. In this and the next sections we shall consider free open SFT. The string coordinates are Fourier-expanded

\footnotetext{
* Our flat metric is $g_{\mu \nu}=\operatorname{diag}(-1,1, \cdots, 1)$. Therefore, a space-like vector has positive norm.

$\dagger$ Although ref. [1] treats also covariant SFT, our conclusion is different from that of ref. [1].
} 
as follows:

$$
\begin{aligned}
& X^{\mu}(\sigma)=x^{\mu}+\sum_{n \geq 1} x_{n}^{\mu} \cos n \sigma, \\
& \Theta(\sigma)=\theta_{0}+\sum_{n \geq 1} \theta_{n} \cos n \sigma \\
& \bar{\Theta}(\sigma)=\sum_{n \geq 1} \bar{\theta}_{n} \sin n \sigma
\end{aligned}
$$

where $\Theta$ and $\bar{\Theta}$ are hermitian. After fixing the stringy local gauge symmetry by adopting the Siegel gauge [7] which imposes the condition that the string field be independent of $\theta_{0}$, the gauge-fixed action of free SFT reads

$$
S=-\frac{1}{2} \int \mathcal{D} Z(\sigma) \Phi^{\dagger}[Z(\sigma)] L \Phi[Z(\sigma)]
$$

where $Z(\sigma)$ denotes the set of string coordinates, $Z(\sigma) \equiv\left(X^{\mu}(\sigma), \Theta^{\prime}(\sigma), \bar{\Theta}(\sigma)\right)$, and $L$ is the kinetic operator (prime denotes $\partial / \partial \sigma)$ :

$$
\begin{aligned}
L & =\pi \int_{0}^{\pi} d \sigma\left\{-g^{\mu \nu} \frac{\delta}{\delta X^{\mu}} \frac{\delta}{\delta X^{\nu}}+g_{\mu \nu} X^{\prime \mu} X^{\prime \nu}-2 i\left(\Theta^{\prime} \bar{\Theta}+\left(\frac{\delta}{\delta \bar{\Theta}}\right)^{\prime} \frac{\delta}{\delta \Theta}\right)\right\} \\
& =-\left(\partial / \partial x^{\mu}\right)^{2}+M^{2}
\end{aligned}
$$

with the (mass) $)^{2}$ operator $M^{2}$ being given in terms of the Fourier components as

$$
M^{2}=2 \sum_{n=1}^{\infty}\left\{-g^{\mu \nu} \frac{\partial}{\partial x_{n}^{\mu}} \frac{\partial}{\partial x_{n}^{\nu}}+\left(\frac{n \pi}{2}\right)^{2} g_{\mu \nu} x_{n}^{\mu} x_{n}^{\nu}+2 i n\left(-\frac{\partial}{\partial \bar{\theta}_{n}} \frac{\partial}{\partial \theta_{n}}+\left(\frac{\pi}{2}\right)^{2} \theta_{n} \bar{\theta}_{n}\right)\right\} .
$$

The present string field $\Phi$ is Grassmann-even and it is hermitian,

$$
\Phi^{\dagger}[Z(\sigma)]=\Phi[Z(\sigma)]
$$

Quantization of free open SFT is carried out by taking the center-of-mass time coordinate $x^{0}$ as the time variable. Then, the string field commutator $[\Phi[Z(\sigma)], \Phi[\widetilde{Z}(\sigma)]]$ is derived from the equal-time canonical commutation relation for $\Phi$ and its conjugate momentum $\Pi=$ $\delta S / \delta \dot{\Phi}=\dot{\Phi}$ together with the equation of motion $L \Phi=0$. In order to perform this procedure precisely, we here make the equivalent calculation based on the local component fields of $\Phi[Z(\sigma)]$. Namely, we expand $\Phi$ in terms of the component fields $\varphi_{A}(x)$ :

$$
\Phi=\sum_{A} \Psi_{A}\left(x_{n}^{\mu}, \theta_{n}, \bar{\theta}_{n}\right) \varphi_{A}(x)
$$

where $\Psi_{A}$ is an eigenfunction of the (mass) ${ }^{2}$ operator (2.6). The concrete expression of $\Psi_{A}$ reads

$$
\Psi_{A}=\prod_{n=1}^{\infty} i^{M_{n}^{0}} h_{M_{n}^{0}}^{(n)}\left(i x_{n}^{0}\right) \cdot \prod_{i=1}^{D-1} h_{M_{n}^{i}}^{(n)}\left(x_{n}^{i}\right) \cdot \psi_{m_{n}, \bar{m}_{n}}\left(\theta_{n}, \bar{\theta}_{n}\right)
$$


where the wave function $h_{M}^{(n)}(x)(M=0,1,2, \cdots)$ for the space-time oscillators $x_{n}^{\mu}$ and the wave function $\psi_{m, \bar{m}}(\theta, \bar{\theta})(m, \bar{m}=0,1)$ for the ghost oscillators $\left(\theta_{n}, \bar{\theta}_{n}\right)$ are given respectively as

$$
h_{M}^{(n)}(x)=\sqrt{\frac{1}{M !} \sqrt{\frac{n}{2}}} H_{M}(\sqrt{\pi n} x) e^{-\pi n x^{2} / 4},
$$

with $H_{M}$ being the Hermite polynomial, and

$$
\begin{aligned}
& \psi_{0,0}(\theta, \bar{\theta})=\frac{1}{\sqrt{\pi}} e^{-i \pi \theta \bar{\theta} / 2}=\frac{1}{\sqrt{\pi}}\left(1-\frac{i \pi}{2} \theta \bar{\theta}\right), \\
& \psi_{1,0}(\theta, \bar{\theta})=\theta e^{-i \pi \theta \bar{\theta} / 2}=\theta, \\
& \psi_{0,1}(\theta, \bar{\theta})=\bar{\theta} e^{-i \pi \theta \bar{\theta} / 2}=\bar{\theta}, \\
& \psi_{1,1}(\theta, \bar{\theta})=\frac{1}{\sqrt{\pi}}(1+i \pi \theta \bar{\theta}) e^{-i \pi \theta \bar{\theta} / 2}=\frac{1}{\sqrt{\pi}}\left(1+\frac{i \pi}{2} \theta \bar{\theta}\right) .
\end{aligned}
$$

Therefore, the index $A$ labeling the oscillator mode is $A=\left\{M_{n}^{\mu}, m_{n}, \bar{m}_{n}\right\}_{n=1,2,3, \cdots}$, and the $(\text { mass })^{2}$ for the state $A$ is given by

$$
M_{A}^{2}=2 \pi \sum_{n=1}^{\infty} n\left(\sum_{\mu=0}^{D-1} M_{n}^{\mu}+m_{n}+\bar{m}_{n}\right)+M_{0}^{2}
$$

where $M_{0}^{2}=-2 \pi$ is the (mass) ${ }^{2}$ of the lightest (tachyon) state. Of course, it is meaningless to discuss causality in a theory containing the tachyon state. However, in the following we pretend that $M_{0}^{2}$ is non-negative since our arguments should apply also to more realistic tachyon-free superstring field theories. It should be understood that the product over $n=1,2, \cdots$ in (2.9) is ordered in such a way that the wave function with a larger $n$ is placed at more right-hand position. Note that each of the factor wave functions in (2.9), in particular, $i^{M_{n}^{0}} h_{M_{n}^{0}}^{(n)}\left(i x_{n}^{0}\right)$ and $\psi_{m_{n}, \bar{m}_{n}}\left(\theta_{n}, \bar{\theta}_{n}\right)$, are hermitian if we regard $\left(x_{n}^{\mu}, \theta_{n}, \bar{\theta}_{n}\right)$ as hermitian.

In order to carry out the quantization procedure, we define the inner-product $\eta_{A B}$ between the wave functions $\Psi_{A}$ and $\Psi_{B}$ by

$$
\eta_{A B}=\prod_{n=1}^{\infty}\left(\int_{-i \infty}^{i \infty} i d x_{n}^{0} \cdot \prod_{i=1}^{D-1} \int_{-\infty}^{\infty} d x_{n}^{i} \cdot i \int d \bar{\theta}_{n} d \theta_{n}\right) \Psi_{A}^{\dagger}\left(x_{n}^{\mu}, \theta_{n}, \bar{\theta}_{n}\right) \Psi_{B}\left(x_{n}^{\mu}, \theta_{n}, \bar{\theta}_{n}\right)
$$

where the integrations over the Grassmann-odd coordinates $\theta_{n}$ and $\bar{\theta}_{n}$ are defined by $\int d \theta_{n} \theta_{n}=$ $\int d \bar{\theta}_{n} \bar{\theta}_{n}=1$. The special treatment for the (negative-norm) $x_{n}^{0}$ oscillator should particularly be explained. First, the hermitian-conjugation $\Psi^{\dagger}$ should be defined by regarding $x_{n}^{0}$ as an hermitian variable. Second, the integration over $x_{n}^{0}$ in (2.13) should be performed in the pure-imaginary direction [9]. The integration measure $\int \mathcal{D} Z(\sigma)$ in the action (2.4) is the same measure as in (2.13) for the oscillator modes multiplied by $\int d^{D} x$ for the center-of-mass coordinate $x^{\mu}$. 
The explicit expression of $\eta_{A B}$ for the wave functions (2.9) is given by

$$
\eta_{A \widetilde{A}}=\prod_{n}(-)^{M_{n}^{0}} \prod_{\mu=0}^{D-1} \delta_{M_{n}^{\mu}, \widetilde{M}_{n}^{\mu}} \cdot \eta_{\left(m_{n}, \bar{m}_{n}\right)\left(\widetilde{m}_{n}, \overline{\tilde{m}}_{n}\right)}
$$

where $\eta_{\left(m_{n}, \bar{m}_{n}\right)\left(\widetilde{m}_{n}, \widetilde{m}_{n}\right)}$ is the inner-product for the ghost oscillator wave functions:

$$
\eta_{(m, \bar{m})(\widetilde{m}, \overline{\tilde{m}})}=i \int d \bar{\theta} d \theta \psi_{m, \bar{m}}^{\dagger} \psi_{\widetilde{m}, \overline{\widetilde{m}}}
$$

For our particular choice of $\psi_{m, \bar{m}}(2.11)$, the non-vanishing component of $\eta_{(m, \bar{m})(\widetilde{m}, \overline{\widetilde{m}})}$ reads

$$
\eta_{(0,0)(0,0)}=1, \quad \eta_{(1,0)(0,1)}=-\eta_{(0,1)(1,0)}=i, \quad \eta_{(1,1)(1,1)}=-1 .
$$

Then, we shall derive the (anti-)commutation relations between the component fields $\varphi_{A}(x)$. Let us define the sign factor $\beta_{A}= \pm$ for the wave function $\Psi_{A}$ by

$$
\Psi_{A}^{\dagger}=\beta_{A} \Psi_{A}
$$

The hermiticity (2.7) implies that $\varphi_{A}^{\dagger}(x)=\beta_{A}(-)^{A} \varphi_{A}(x)$, and the action (2.4) is expressed in terms of $\varphi_{A}$ as

$$
S=\frac{1}{2} \sum_{A, B} \beta_{A}(-)^{A} \eta_{A B} \int d^{D} x \varphi_{A}(x)\left(\partial^{2}-M_{A}^{2}\right) \varphi_{B}(x) .
$$

Imposing the equal center-of-mass time canonical (anti-)commutation relation,

$$
\left[\varphi_{A}(x), \pi_{B}(\widetilde{x})\right\}_{x^{0}=\widetilde{x}^{0}}=i \delta_{A B} \delta^{D-1}(\boldsymbol{x}-\widetilde{\boldsymbol{x}}),
$$

with $\pi_{A}(x) \equiv \delta S / \delta \dot{\varphi}_{A}(x)=\beta_{A}(-)^{A} \sum_{B} \eta_{A B} \dot{\varphi}_{B}(x)$, the (anti-)commutation relation between $\varphi$ 's with general space-time coordinates is given by

$$
\left[\varphi_{A}(x), \varphi_{B}(\widetilde{x})\right\}=\beta_{A}(-)^{A}\left(\eta^{-1}\right)_{B A} \cdot i \Delta\left(x-\widetilde{x} ; M_{A}^{2}\right),
$$

using the invariant function $\Delta$ :

$$
i \Delta\left(x ; M^{2}\right)=\int \frac{d^{D} k}{(2 \pi)^{D-1}} \operatorname{sgn}\left(k_{0}\right) \delta\left(k^{2}+M^{2}\right) e^{i k \cdot x} .
$$

Having obtained the commutator for the local component fields, the commutation relation for the original string field $\Phi$ is calculated from eqs. (2.7), (2.8) and (2.20) to be given by

$$
[\Phi[Z], \Phi[\widetilde{Z}]]=\sum_{A B} \Psi_{A}^{\dagger}[Z]\left(\eta^{-1}\right)_{B A} \Psi_{B}[\widetilde{Z}] \cdot i \Delta\left(x-\widetilde{x} ; M_{A}^{2}\right) .
$$

\footnotetext{
$\ddagger(-)^{A}=+(-)$ if $\Psi_{A}$ is Grassmann-even (odd).

$\S\left[\varphi_{A}, \pi_{B}\right\} \equiv \varphi_{A} \pi_{B}-(-)^{A B} \pi_{B} \varphi_{A}$.
} 
Our final task in this section is to carry out the summation over the string modes $A$ and $B$ in eq. (2.22). For this purpose, we make use of the integral expression of the delta function, $\delta\left(k^{2}+M^{2}\right)=\int_{-\infty}^{\infty} d \tau / 2 \pi \exp \left\{i \tau\left(k^{2}+M^{2}\right)\right\}$, to rewrite the invariant function (2.21) as a double integral with respect to $\tau$ and $u=\left(2 \tau / x^{0}\right) k_{0}$ :

$$
i \Delta\left(x ; M^{2}\right)=i x^{0} \int_{-1}^{1} d u \int_{-\infty}^{\infty} d \tau\left(\frac{i}{4 \pi \tau}\right)^{(D+1) / 2} \exp \left\{-\frac{i}{4 \tau}\left[\boldsymbol{x}^{2}-\left(1-u^{2}\right)\left(x^{0}\right)^{2}\right]+i \tau M^{2}\right\} .
$$

Then, the summation over $A$ and $B$ in eq. (2.22) is carried out using the formula for $h_{M}^{(n)}(x)$,

$$
\sum_{M=0}^{\infty} h_{M}^{(n)}(x) h_{M}^{(n)}(\widetilde{x}) e^{i \tau \cdot 2 \pi n M}=\sqrt{\frac{n}{2\left(1-e^{4 \pi i n \tau}\right)}} \exp \left\{\frac{\pi n}{4 i \sin (2 \pi n \tau)}\left[\left(x^{2}+\widetilde{x}^{2}\right) \cos (2 \pi n \tau)-2 x \widetilde{x}\right]\right\},
$$

and a similar one for $\psi_{m, \bar{m}}$,

$$
\begin{aligned}
& \sum_{m, \bar{m}} \sum_{\widetilde{m}, \overline{\widetilde{m}}} \psi_{m, \bar{m}}^{\dagger}(\theta, \bar{\theta})\left(\eta^{-1}\right)_{(\widetilde{m}, \overline{\tilde{m}})(m, \bar{m})} \psi_{\widetilde{m}, \overline{\tilde{m}}}(\widetilde{\theta}, \overline{\widetilde{\theta}}) e^{i \tau \cdot 2 \pi n(m+\bar{m})} \\
& =\psi_{0,0}^{\dagger} \psi_{0,0}-i\left(\psi_{1,0}^{\dagger} \psi_{0,1}-\psi_{0,1}^{\dagger} \psi_{1,0}\right) e^{2 \pi i n \tau}-\psi_{1,1}^{\dagger} \psi_{1,1} e^{4 \pi i n \tau} \\
& =\frac{1-e^{4 \pi i n \tau}}{\pi} \exp \left\{\frac{\pi}{2 \sin (2 \pi n \tau)}[(\theta \bar{\theta}+\widetilde{\theta} \overline{\tilde{\theta}}) \cos (2 \pi n \tau)+\theta \overline{\tilde{\theta}}+\widetilde{\theta} \bar{\theta}]\right\} .
\end{aligned}
$$

We obtain the following master expression of the string field commutator:

$$
\begin{aligned}
& {[\Phi[Z], \Phi[\widetilde{Z}]]=i \Delta x^{0} \int_{-1}^{1} d u \int_{-\infty}^{\infty} d \tau\left(\frac{i}{4 \pi \tau}\right)^{(D+1) / 2}} \\
& \times \exp \left\{-\frac{i}{4 \tau}\left[(\Delta \boldsymbol{x})^{2}-\left(1-u^{2}\right)\left(\Delta x^{0}\right)^{2}\right]+i \tau M_{0}^{2}\right\} \\
& \times \prod_{n=1}^{\infty}\left(\frac{n}{2}\right)^{D / 2} \frac{1}{\pi\left(1-e^{4 \pi i n \tau}\right)^{(D-2) / 2}} \exp \left\{\frac { \pi } { 4 i \operatorname { s i n } ( 2 \pi n \tau ) } \left(n\left[\left(x_{n}^{2}+\widetilde{x}_{n}^{2}\right) \cos (2 \pi n \tau)-2 x_{n} \cdot \widetilde{x}_{n}\right]\right.\right. \\
& \left.\left.\quad+2 i\left[\left(\theta_{n} \bar{\theta}_{n}+\widetilde{\theta}_{n} \overline{\widetilde{\theta}}_{n}\right) \cos (2 \pi n \tau)+\theta_{n} \overline{\widetilde{\theta}}_{n}+\widetilde{\theta}_{n} \bar{\theta}_{n}\right]\right)\right\},
\end{aligned}
$$

with $\Delta x^{\mu}=x^{\mu}-\widetilde{x}^{\mu}$ and $x_{n}^{2}=g_{\mu \nu} x_{n}^{\mu} x_{n}^{\nu}$.

Here, we have derived the string field commutator starting from the equal-time canonical (anti-)commutation relations for the component fields. However, looking back the above calculation, the equal-time canonical commutation relation for the string field $\Phi$ and its conjugate $\Pi=\dot{\Phi}$ is seen to be given by

$$
[\Phi[Z], \dot{\Phi}[\widetilde{Z}]]_{x^{0}=\widetilde{x}^{0}}=i \delta^{D-1}(\boldsymbol{x}-\widetilde{\boldsymbol{x}}) \prod_{n=1}^{\infty} \delta\left(i x_{n}^{0}-i \widetilde{x}_{n}^{0}\right) \delta^{D-1}\left(\boldsymbol{x}_{n}-\widetilde{\boldsymbol{x}}_{n}\right) \cdot(-i) \delta\left(\theta_{n}+\widetilde{\theta}_{n}\right) \delta\left(\bar{\theta}_{n}+\overline{\widetilde{\theta}}_{n}\right) .
$$




\section{Causality in covariant open SFT}

We would like to argue that the commutator (2.26) vanishes if the space-time string coordinates $X^{\mu}(\sigma)$ and $\widetilde{X}^{\mu}(\sigma)$ satisfy the condition (1.1). For this purpose, we need two kinds of regularizations for eq. (2.26). One is for the infinite product over the oscillator mode number $n$ in eq. (2.26). As this regularization we shall adopt either cutting off the mode number $n$ at $n=N$, or smearing the string field $\Phi$ with smooth test functions $g^{(n)}\left(x_{n}^{\mu}, \theta_{n}, \bar{\theta}_{n}\right)$ for the higher modes $n \geq N+1$ as was done in ref. [5] for the light-cone gauge SFT. Here we shall first adopt the cutoff method for $n$ and replace the infinite product $\prod_{n=1}^{\infty}$ in eq. (2.26) with $\prod_{n=1}^{N}$. The other regularization we need is for the singularities of the $\tau$-integrand of eq. (22.26), which we shall explain below.

Let us consider the $\tau$-integration in eq. (2.26) along the real axis for a fixed $u$ in the range $|u| \leq 1$. Since the integrand is singular at the origin $\tau=0$ and at $\tau=\tau_{(k / n)} \equiv k / 2 n(n=$ $1,2, \cdots, N, k= \pm 1, \pm 2, \cdots)$, we regularize the integration by excluding from the integration region $(-\infty, \infty)$ the neighborhoods of these singular points, $|\tau|<\eta$ and $\left|\tau-\tau_{(k / n)}\right|<\eta$, and then take the limit $\eta \rightarrow 0$. To evaluate this $\tau$-integration, we consider the contour of Fig. 1, which is obtained by adding small semicircles with radius $\eta, C_{\eta}^{(0)}$ at the origin and $C_{\eta}^{(k / n)}$ at $\tau_{(k / n)}$, and a large one $C_{R}$ with radius $R$ to the regularized path (cutoff at $|\tau|=R$ ) along the real axis. Since the integrand is non-singular inside the contour, the original $\tau$-integration of eq. (2.26) along the real axis and hence the string field commutator vanish if the contributions from the semicircles, $C_{\eta}^{(0)}, C_{\eta}^{(k / n)}$ and $C_{R}$, vanish in the limits $\eta \rightarrow 0$ and $R \rightarrow \infty$.

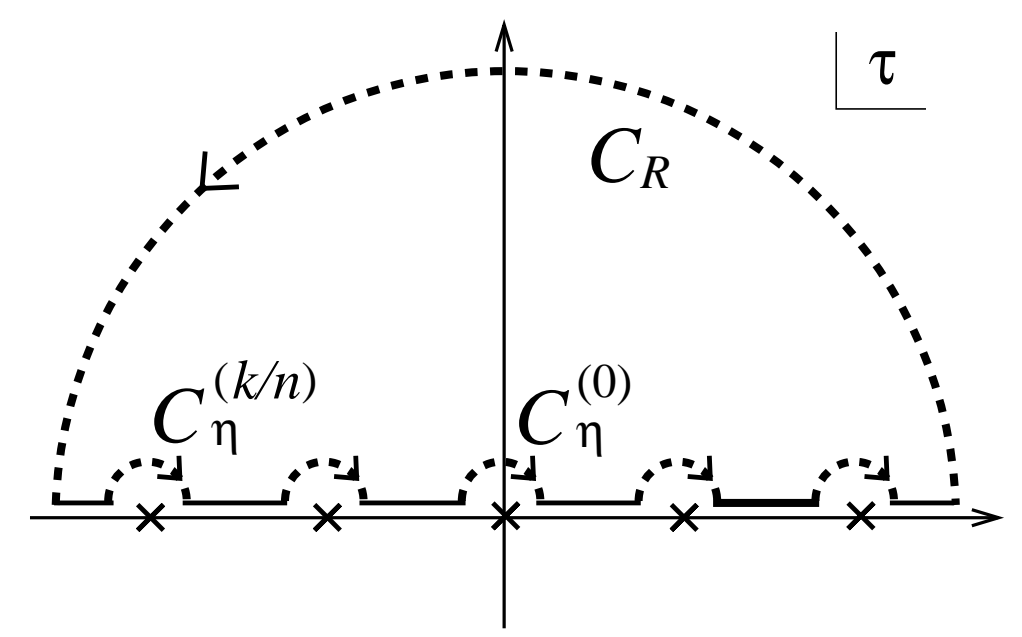

Figure 1: The $\tau$-integration contour. The crosses indicate the singularities. 
In order to discuss the conditions for the contributions from the semicircles to vanish, we should first understand that the part of the exponent in (2.26) containing the Grassmann-odd variables $\left(\theta_{n}, \bar{\theta}_{n}\right)$ and $\left(\widetilde{\theta}_{n}, \overline{\tilde{\theta}}_{n}\right)$ has little effect on the following arguments. This is because the last exponentiated expression of eq. (2.25) which is apparently singular at $\tau=\tau_{(k / n)}$ is in fact the sum of three terms proportional to $e^{2 m \pi i n \tau}(m=0,1,2)$ as the second expression of eq. (2.25) shows. Recalling that the product over $n$ in (2.26) is now a finite one, we see that the Grassmann-odd variable parts only affect the exponent $P$ in eq. (3.1) below.

It is easily seen that the contribution from $C_{R}$ vanishes in the limit $R \rightarrow \infty$ if we have $M_{0}^{2} \geq 0$, which we assume true as explained in the previous section. Next, let us consider $C_{\eta}^{(0)}$ at the origin. Taking the above argument for the terms containing the Grassmannodd variables into account, we see that each term of the $\tau$-integrand in (2.26) obtained by expanding with respect to the Grassmann-odd variables behaves near the origin $\tau \sim 0$ as

$$
\left(\frac{1}{\tau}\right)^{P / 2} \exp \left\{-\frac{i}{4 \tau}\left[(\Delta \boldsymbol{x})^{2}-\left(1-u^{2}\right)\left(\Delta x^{0}\right)^{2}+\frac{1}{2} \sum_{n=1}^{N}\left(\Delta x_{n}^{\mu}\right)^{2}\right]\right\}
$$

where we have $\Delta x_{n}^{\mu}=x_{n}^{\mu}-\widetilde{x}_{n}^{\mu}$, and $P$ is some integer. Therefore, the contribution from $C_{\eta}^{(0)}$ vanishes for every $u(|u| \leq 1)$ if the condition,

$$
(\Delta \boldsymbol{x})^{2}-\left(\Delta x^{0}\right)^{2}+\frac{1}{2} \sum_{n=1}^{N}\left(\Delta x_{n}^{\mu}\right)^{2}>0
$$

is satisfied. Taking the limit $N \rightarrow \infty$, we obtain the condition (1.1).

The estimate of the contribution from $C_{\eta}^{(k / n)}$ is quite similar. Here, complication arises due to the fact that different $(n, k)$ 's with coinciding ratio $k / n$ give the same $\tau_{(k / n)}$. In any case, we find that the contribution from $C_{\eta}^{(k / n)}$ 's vanish in the limit $\eta \rightarrow 0$ if the condition,

$$
\left(x_{n} \pm \widetilde{x}_{n}\right)^{2} \geq 0,
$$

is satisfied for every $n$. Implication of this condition (3.3) will soon be explained below.

Although the condition (1.1) for the covariant SFT is apparently the same as the one in the light-cone gauge formulation [1, 5], their expressions in terms of the oscillator modes $x_{n}^{\mu}$ are different: the condition (1.1) in the light-cone gauge contains only the transverse oscillators while (1.1) for the covariant formulation depends on $x_{n}^{\mu}$ for all $\mu$. In particular, the timelike oscillator modes $x_{n}^{0}$ contribute to (1.1) with negative sign; $-\left(\Delta x_{n}^{0}\right)^{2}$. In the light-cone gauge formulation, if the center-of-mass coordinates of two strings are space-like separated, the condition (1.1) is automatically satisfied. On the other hand, one might think that this property no longer holds in the covariant formulation due to the negative sign terms, $-\left(\Delta x_{n}^{0}\right)^{2}$. 
However, as explained in Sec. 2 the time-like oscillators $x_{n}^{0}$ take pure-imaginary values. (In the first quantization language, $x_{n}^{0}$ is an hermitian operator but its eigenvalues are pure-imaginary [9].) This implies that, in the covariant formulation also, the string field commutator vanishes if the center-of-mass string coordinates are space-like separated. Therefore, although the RHS of eq. (2.26) is multiplied by $\Delta x^{0}$, we do not need to take care of the condition $\Delta x^{0}=0$ as another sufficient condition for the string field commutator to vanish. The fact that the timelike oscillators $x_{n}^{0}$ take pure-imaginary values also implies that the condition (3.3) is always satisfied and hence may completely be forgotten. Summarizing, the commutator of free open string fields vanish if the condition (1.1) is satisfied.

The above arguments can be repeated to obtain the same conclusion if we adopt the smearing regularization [5] instead of the cutoff one for the mode number $n$. We do not present the details here but only mention that the argument of ref. [5] applies also to the present ghost coordinate parts. The point is that the ghost coordinates part in (2.26) reduces to the delta function in the limit $\tau \rightarrow 0$ :

$$
-4 i n \tau \exp \left\{\frac{1}{4 n \tau}\left(\theta_{n}+\widetilde{\theta}_{n}\right)\left(\bar{\theta}_{n}+\overline{\widetilde{\theta}}_{n}\right)\right\} \rightarrow-i \delta\left(\theta_{n}+\widetilde{\theta}_{n}\right) \delta\left(\bar{\theta}_{n}+\overline{\widetilde{\theta}}_{n}\right) .
$$

\section{Causality in closed SFT}

The arguments of the previous sections for open SFT can be extended to the closed SFT case. Here we briefly explain the points particular to closed SFT. The string coordinate of the closed string is Fourier expanded into both the cosine and the sine modes as

$$
X^{\mu}(\sigma)=x^{\mu}+\sum_{n \geq 1}\left(x_{C n}^{\mu} \cos n \sigma+x_{S n}^{\mu} \sin n \sigma\right)
$$

for the space-time coordinate $X^{\mu}(\sigma)$ and similarly for the ghost coordinates. An important point for closed SFT is that the string field $\Phi[Z(\sigma)]$ is subject to the constraint that it be invariant under the rigid shift of the parameter $\sigma$, namely,

$$
\Phi[Z(\sigma+\theta)]=\Phi[Z(\sigma)]
$$

for an arbitrary $\theta$. To obtain the correct closed string field commutator which takes into account the constraint (4.2), we have to make the following modifications on eq. (2.26). First, noting that, under the shift of $\sigma$ in (4.1) by an amount $\theta$, the Fourier coefficients $\left(x_{C n}^{\mu}, x_{S n}^{\mu}\right)$ is transformed as

$$
\left(\begin{array}{c}
x_{C n}^{\mu} \\
x_{S n}^{\mu}
\end{array}\right) \rightarrow\left(\begin{array}{c}
x_{C}(\theta)_{n}^{\mu} \\
x_{S}(\theta)_{n}^{\mu}
\end{array}\right)=\left(\begin{array}{cc}
\cos n \theta & \sin n \theta \\
-\sin n \theta & \cos n \theta
\end{array}\right)\left(\begin{array}{c}
x_{C n}^{\mu} \\
x_{S n}^{\mu}
\end{array}\right)
$$


we replace the quantity $\left(x_{n}^{2}+\widetilde{x}_{n}^{2}\right) \cos (2 \pi n \tau)-2 x_{n} \cdot \widetilde{x}_{n}$ in the exponent of eq. (2.26) by

$$
\sum_{\alpha=C, S}\left\{\left(\left[x_{\alpha}(\theta)_{n}\right]^{2}+\left[\widetilde{x}_{\alpha}(\widetilde{\theta})_{n}\right]^{2}\right) \cos (2 \pi n \tau)-2 x_{\alpha}(\theta)_{n} \cdot \widetilde{x}_{\alpha}(\widetilde{\theta})_{n}\right\}
$$

A similar replacement is necessary also for the ghost coordinates. Then, we have to carry out the integrations over $\theta$ and $\tilde{\theta}: \int_{0}^{2 \pi} d \theta / 2 \pi \int_{0}^{2 \pi} d \tilde{\theta} / 2 \pi$, which effects the projection of the string field into the subspace satisfying the condition (4.2). Repeating the arguments of the previous section, we see that the (sufficient) condition for the closed string field commutator $[\Phi[Z], \Phi[\widetilde{Z}]]$ to vanish is that the inequality,

$$
(\Delta \boldsymbol{x})^{2}-\left(1-u^{2}\right)\left(\Delta x^{0}\right)^{2}+\frac{1}{2} \sum_{n=1}^{N} \sum_{\alpha=C, S}\left(\left[x_{\alpha}(\theta)_{n}\right]^{2}+\left[\widetilde{x}_{\alpha}(\widetilde{\theta})_{n}\right]^{2}-2 x_{\alpha}(\theta)_{n} \cdot \widetilde{x}_{\alpha}(\widetilde{\theta})_{n}\right)>0
$$

holds for an arbitrary $u \in[-1,1]$ and arbitrary $\theta, \widetilde{\theta} \in[0,2 \pi]$. This implies that the condition (1.1) should be replaced for closed SFT by (1.2). The condition (1.2) is indeed invariant under independent shifts of the $\sigma$ parameters of the two string coordinates.

\section{Summary}

In this paper, we have analyzed causality in free covariant SFT. We obtained, as a sufficient condition for the string field commutator to vanish, (1.1) for open SFT and (1.2) for closed SFT. Compared with the light-cone gauge SFT, the string field in covariant SFT depends additionally on the ghost coordinates and the time-like oscillator modes of $X^{0}(\sigma)$. We found that the ghost coordinates do not enter the analysis of causality. As for the time-like oscillator modes, the fact that $x_{n}^{0}$ take pure-imaginary values was crucial for the commutability condition to be given simply by (1.1) or (1.2).

From the view point of the component fields, the string field commutator should vanish if the center-of-mass string coordinates are space-like separated. The conditions (1.1) and (1.2) imply that the commutative region is "enlarged" as a result of the string mode summation: the commutator vanishes if the center-of-mass coordinates are space-like separated, however the commutator can vanish even if the separation of center-of-mass coordinates is time-like due to the oscillator mode terms in (1.1) and (1.2).

\section{Acknowledgments}

We would like to thank S. Yahikozawa for valuable discussions. 


\section{References}

[1] E. Martinec, Class. Quant. Grav. 10 (1993) L187.

[2] S. Mandelstam, Nucl. Phys. B64 (1973) 205; B83 (1974) 413.

[3] E. Cremmer and J.-L. Gervais, Nucl. Phys. B76 (1974) 209; B90 (1975) 410.

[4] M. Kaku and K. Kikkawa, Phys. Rev. D10 (1974) 1110; D10 (1974) 1823.

[5] D. A. Lowe, Phys. Lett. 326B (1994) 223.

[6] D. A. Lowe, L. Susskind and J. Uglum, Phys. Lett. 327B (1994) 226.

[7] W. Siegel, Phys. Lett. 149B (1984) 157; 149B (1984) 162; 151B (1985) 391; 151B (1985) 396.

[8] W. Siegel and B. Zwiebach, Nucl. Phys. B263 (1986) 105; K. Itoh, T. Kugo, H. Kunitomo and H. Ooguri, Prog. Theor. Phys. 75 (1986) 162; T. Banks and M. E. Peskin, Nucl. Phys. B264 (1986) 513.

[9] H. Arisue, T. Fujiwara, T. Inoue, and K. Ogawa, J. Math. Phys. 22 (1981) 2055. 